\title{
X-Ray Variability of the O Star $\zeta$ Puppis
}

\author{
Thomas W. Berghöfer ${ }^{1,2}$ \\ 1 Space Sciences Laboratory, University of California, Berkeley, CA 94720, USA \\ 2 Hamburger Sternwarte, Gojenbergsweg 112, D-21029 Hamburg, Germany
}

\section{X-ray emission of $\mathrm{O}$ stars}

X-ray surveys carried out with the Einstein Observatory (Chlebowski et al. 1989) and ROSAT (Berghöfer et al. 1996) have shown that all O stars are soft $\mathrm{X}$-ray emitters. Since O star winds are opaque at soft X-ray energies the stars or their photospheres cannot be the origin of the observed X-ray emission, thus, this emission must be produced in their stellar winds. Obviously, the $\mathrm{X}$-ray emission is connected to dynamical processes present in the winds of $\mathrm{O}$ stars; steady-state computations for $\mathrm{O}$ star winds which are able to explain many of the observational features cannot predict any X-ray emission.

Lucy \& White (1980) suggested the presence of hot gas in the stellar winds which is produced in shocks developing from the growth of instabilities in the winds; supersonic wind flows in $\mathrm{O}$ stars are known to be intrinsically unstable. Numerical simulations confirmed this scenario. However, so far these simulations are limited to one or two dimensions and are not able to explain for instance the observed X-ray luminosity of $\mathrm{O}$ stars.

Further support for a model of shock-heated gas in the winds of $\mathrm{O}$ stars came from the analysis of the ROSAT PSPC spectrum of $\zeta$ Pup (O4Iaf). This spectrum demonstrates that the X-ray emission is "self-absorbed" by the stellar wind. Based on a detailed modelling of the X-ray opacity and an assumed "uniform" distribution of X-ray sources in the stellar wind, Hillier et al. (1993) were able to explain the observed spectrum of $\zeta$ Pup.

On the other hand, however, shocks should statistically occur and the total number of visible shocks and their X-ray output should be time variable. $\mathrm{X}$-ray variability studies provide the only method to study the dynamical processes involved in the $\mathrm{X}$-ray production in $\mathrm{O}$ star winds and to derive important input parameters needed for model simulations (e.g., occurrence rates and cooling times of shocks). As a result, long term variability studies by Berghöfer \& Schmitt (1994a, 1994b, 1995) demonstrated that X-ray variability on all observable time scales is generally not common for $\mathrm{O}$ stars. So far, only for the $\mathrm{O}$ supergiant $\zeta$ Orionis a moderate increase in $\mathrm{X}$-ray count rate has been found (Berghöfer \& Schmitt 1994c). Here we present results obtained for the prototype O star $\zeta$ Pup. 


\section{X-ray and wind variability of $\zeta$ Pup}

$\zeta$ Pup is one of the most studied O stars in the sky. Variability has been reported for this star on different time scales. A $5 \mathrm{~d}$ period has been attributed to rotational modulation. Several authors found evidence for periodic line profile variations on time scales between 2 and $8 \mathrm{~h}$ at different epochs. These are attributed to non-radial pulsations (NRP) of the photosphere. A reoccurrence time of $19.2 \mathrm{~h}$ for the so-called discrete absorption components (DAC) in UV wind lines has been detected during the IUE MEGA campaign. Simultaneous ROSAT and $\mathrm{H} \alpha$ observations provided evidence for correlated variability in the X-ray and $\mathrm{H} \alpha$ emission of $\zeta$ Pup (Berghöfer et al. 1996). The period of $16.7 \mathrm{~h}$ detected in both data sets has been attributed to a periodic modulation of the wind density at the base of the wind which extends out to the X-ray emitting regions in the wind.

To further investigate these small but significant periodic variations in the X-ray flux of $\zeta$ Pup we obtained a long observation with the BeppoSAX $\mathrm{X}$-ray satellite. In order to demonstrate the long term stability of the X-ray flux of $\zeta$ Pup we show in Fig. 1 the long-term X-ray light curve of $\zeta$ Pup in the energy band 0.9-2.0 keV. Together with the BeppoSAX LECS observation we show data points obtained with the Einstein Observatory, ROSAT PSPC, and ASCA SIS; all count rates were converted to ROSAT PSPC counts/s taking into account the effective areas of the different detectors. As can be seen the X-ray flux of $\zeta$ Pup is constant over a time scale of about 16 years, the deviations from the best fit constant model (dashed line in Fig. 1) are small.

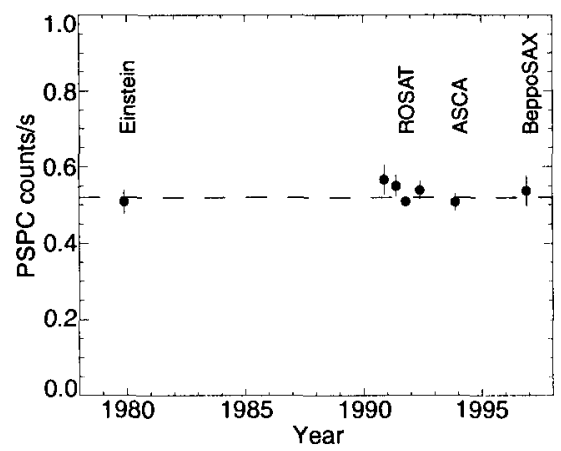

Fig. 1. Long term X-ray light curve of $\zeta$ Pup in the energy band $0.9-2.0 \mathrm{keV}$

A detailed analysis of the $\zeta$ Pup data obtained with BeppoSAX will be published elsewhere. Here we provide first results of a timing analysis. Employing the same methods described in Berghöfer et al. (1996) we carried out a search for periodic variations in the BeppoSAX observations of $\zeta$ Pup. 
Above $0.9 \mathrm{keV}$ both data sets obtained with the LECS and MECS detectors show a period of $15 \mathrm{~h}$. As an example we show in Fig. 2 the BeppoSAX LECS (0.9-3.0 keV) light curve when folded with a $15 \mathrm{~h}$ period. The solid curve shows the best fit for a sinusoidal model.

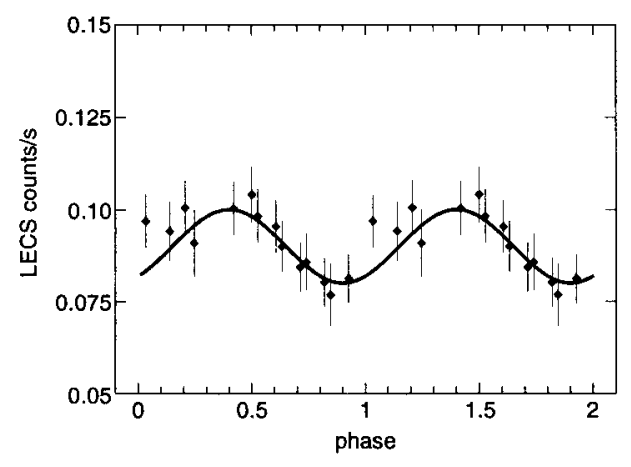

Fig. 2. BeppoSAX light curve of $\zeta$ Pup in the energy band 0.9-3.0 keV when folded with a $15 \mathrm{~h}$ period. The solid curve shows the best fit sinusoidal model.

The BeppoSAX observations of $\zeta$ Pup provide evidence for a $15 \mathrm{~h}$ period in the X-ray flux of this star. This period is significantly different to the 16.7 $h$ period detected in the ROSAT observations 5 years before. However, both periods are of the same order as the $19.2 \mathrm{~h}$ reoccurrence time of DACs observed in 1995. This strongly supports the idea of a quasi-periodic variability of the wind of $\zeta$ Pup triggered by NRPs. The observed modulation of the wind density also provides a perfect trigger mechanisms for the unstable wind to form shocks and hence produce the X-ray emitting hot gas.

T.W.B. acknowledges the support from the Alexander von Humboldt Stiftung $(\mathrm{AvH})$ by a Feodor-Lynen Fellowship.

\section{References}

Berghöfer, T. W., Schmitt, J. H. M. M. 1994a, A\&A 290, 435

Berghöfer, T. W., Schmitt, J. H. M. M. 1994b, ROSAT X-ray light curves of earlytype stars, In: Astrophys. Space Sci. 221, 309

Berghöfer, T. W., Schmitt, J. H. M. M. 1994c, Science 265, 1689

Berghöfer, T. W., Schmitt, J. H. M. M. 1995, Long term X-ray variability studies of OB-type stars, In: Adv. Space Res. Vol. 16, No. 3, p. 163

Berghöfer, T. W., Schmitt, J. H. M. M., Cassinelli, J. P. 1996, A\&AS 118, 481

Berghöfer, T. W., Baade, D., Schmitt, J. H. M. M., et al. 1996, A\&A, 306, 899

Chlebowski, T., Harnden, F. R., Jr., Sciortino, S. 1989, ApJ, 341, 427

Hillier, D. J., Kudritzki, R.-P., Pauldrach, A. W. A., et al. 1993, A\&A, 276, 117

Lucy, L.B., White, R.L. 1980, ApJ, 241, 300 